\title{
In vitro expansion of pancreatic islet clusters facilitated by hormones and chemicals
}

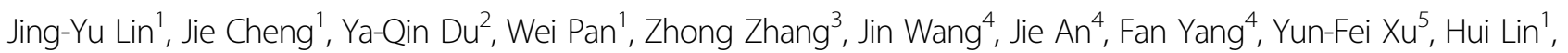
Wen-Tao An ${ }^{1}$, Jia Wang ${ }^{6}$, Zhao Yang ${ }^{6}$, Ren-Jie Chai ${ }^{3}$, Xue-Ying Sha ${ }^{2}$, Hui-Li Hu ${ }^{7}$, Jin-Peng Sun ${ }^{2,6}$ and Xiao Yu ${ }^{1}$

\begin{abstract}
Tissue regeneration, such as pancreatic islet tissue propagation in vitro, could serve as a promising strategy for diabetes therapy and personalised drug testing. However, such a strategy has not been realised yet. Propagation could be divided into two steps, in vitro expansion and repeated passaging. Even the first step of the in vitro islet expansion has not been achieved to date. Here, we describe a method that enables the expansion of islet clusters isolated from pregnant mice or wild-type rats by employing a combination of specific regeneration factors and chemical compounds in vitro. The expanded islet clusters expressed insulin, glucagon and somatostatin, which are markers corresponding to pancreatic $\beta$ cells, a cells and $\delta$ cells, respectively. These different types of cells grouped together, were spatially organised and functioned similarly to primary islets. Further mechanistic analysis revealed that forskolin in our recipe contributed to renewal and regeneration, whereas exendin-4 was essential for preserving islet cell identity. Our results provide a novel method for the in vitro expansion of islet clusters, which is an important step forward in developing future protocols and media used for islet tissue propagation in vitro. Such method is important for future regenerative diabetes therapies and personalised medicines using large amounts of pancreatic islets derived from the same person.
\end{abstract}

\section{Introduction}

Diabetes is characterised by insulin resistance and dysfunctional insulin secretion. Recent progress in the generation of functional insulin-producing pancreatic $\beta$ cells from stem cells differentiation or transdifferentiation in vitro has shed light on cell-replacement therapies for the treatment of diabetes ${ }^{1-4}$. However, compared to tissue/organ replacement therapy derived from somatic islets of the same person for curing diabetes ${ }^{5,6}$, these methods still have the following deficits: (1) The function

Correspondence: Hui-Li Hu (huhuili@sdu.edu.cn) or

Jin-Peng Sun (sunjinpeng@bjmu.edu.cn) or Xiao Yu (yuxiao@sdu.edu.cn)

${ }^{1}$ Key Laboratory Experimental Teratology of the Ministry of Education and

Department of Physiology and Pathophysiology, School of Basic Medical

Sciences, Shandong University, 250012 Jinan, Shandong, China

${ }^{2}$ Department of Physiology and Pathophysiology, School of Basic Medical

Sciences, Peking University, Key Laboratory of Molecular Cardiovascular

Science, Ministry of Education, 100191 Beijing, China

Full list of author information is available at the end of the article.

These authors contributed equally: Jing-Yu Lin, Jie Cheng, Ya-Qin Du, Wei Pan of a tissue, such as pancreatic islets, cannot be fully substituted by a single $\beta$ cell type. Islets contain at least three main cell types, and together, they contribute the functional integrity adaptations to responses to different physiological changes ${ }^{7-10}$. In fact, recent reports indicate the essential roles of the islet circuit in maintaining glucose metabolism homoeostasis ${ }^{7,10}$. (2) The identity of the $\beta$ cells generated from transdifferentiation or stem cells is not defined, and their functions are not guaranteed. These cells normally have different gene expression patterns or certain mutations compared to somatic $\beta$ cells. (3) Some of the $\beta$-like cells generated from stem cells may still have pluripotency potential, which may correlate to a risk of tumour development. (4) Finally, these cells may elicit unwanted immune responses.

Ideally, propagating islets isolated from the same patient to the desired number and then transplanting them into the patient is a promising regenerative strategy for diabetes therapy with benefits that stem cell- or

\section{(c) The Author(s) 2020}

(c) Open Access This article is licensed under a Creative Commons Attribution 4.0 International License, which permits use, sharing, adaptation, distribution and reproduction cc) in any medium or format, as long as you give appropriate credit to the original author(s) and the source, provide a link to the Creative Commons license, and indicate if changes were made. The images or other third party material in this article are included in the article's Creative Commons license, unless indicated otherwise in a credit line to the material. If material is not included in the article's Creative Commons license and your intended use is not permitted by statutory regulation or exceeds the permitted use, you will need to obtain permission directly from the copyright holder. To view a copy of this license, visit http://creativecommons.org/licenses/by/4.0/. 


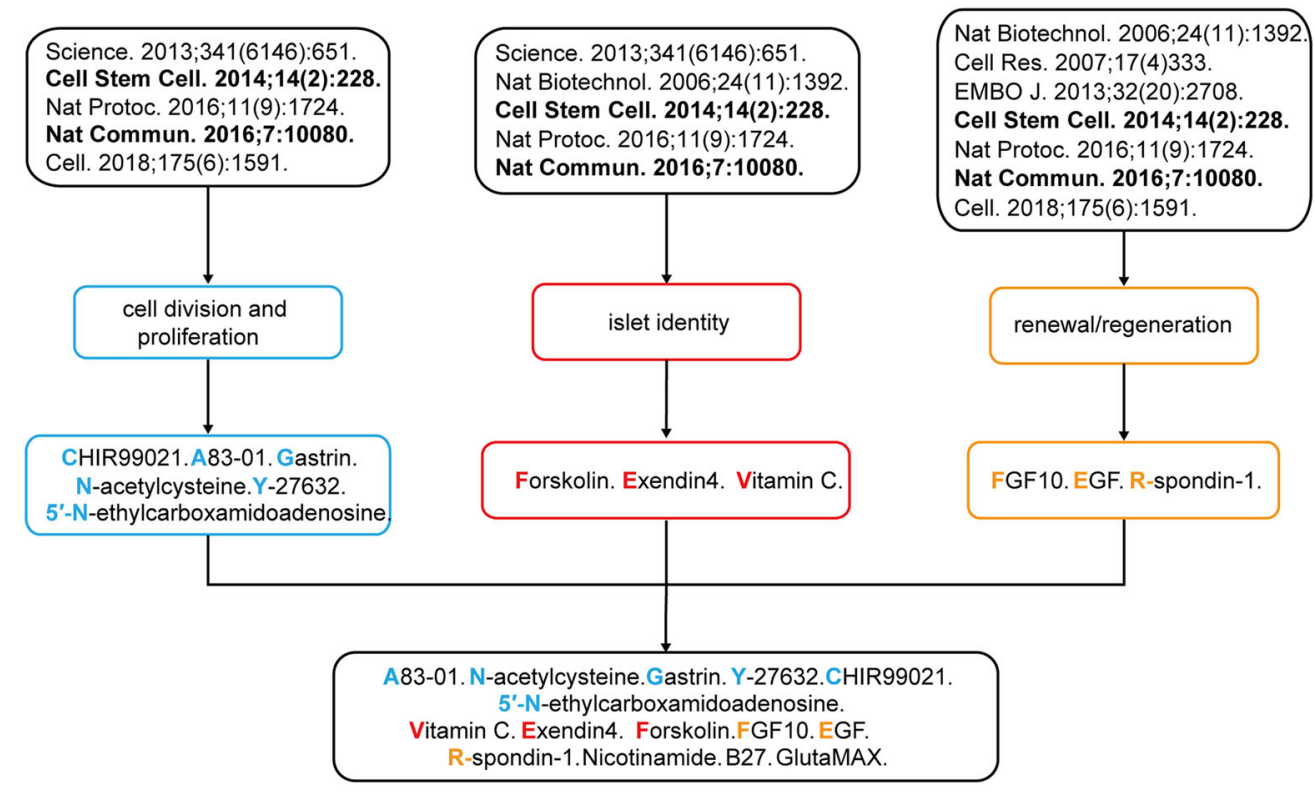

Fig. 1 Flowchart for the development of the medium recipe used for pancreatic islet expansion. The chemicals and hormones used in previous studies for stem cell expansion, differentiation and pancreatic islet cell conversion (upper panel) are classified by their reported function related to cell division and proliferation, islet identity, and cell renewal and regeneration (middle panel). The common factors are summarised and combined for the formulation of the medium recipe used for in vitro pancreatic islet cluster expansion.

transdifferentiation-based cell-replacement treatment cannot match. This method will minimise the immune response, decrease the identity difference and result in a better ability to adapt to different growth niches after transplantation. In addition, the organoids generated from pancreatic islets propagated from patients can be used for drug testing because they mostly model composition, architecture and function of primary tissues. Recently, significant progress has been made that somatic intestinal, gastric, colonal, hepatic and pancreatic ducts were cultured and propagated to organoids in vitro ${ }^{11-19}$, which lays the foundation for future regenerative therapy. However, these organoids mostly exhibit epithelial properties and the methods to generate such organoids cannot be used directly for endocrine cells. Thus, the in vitro propagation of functional endocrine organoids especially islets, is still required to be established.

Propagation of islets could be divided by two processes: (1) expansion of the islet cells in vitro; (2) propagating the expanded islet cells by multiple passages. Here, we reported a method for $3 \mathrm{D}$ culture with a novel special medium recipe that enabled the in vitro expansion of pancreatic islet clusters isolated from pregnant mice or wild-type rats. Further mechanistic analysis suggested that forskolin (FSK) in the recipe is required for maintaining cell renewal and regeneration, whereas exendin-4 is essential for preserving islet identity. Therefore, our study identified a method allowing an in vitro expansion of the pancreatic islet clusters, which could be a key step for propagating patient-specific pancreatic islets for future regenerative diabetes therapy.

\section{Results}

Recipe for the in vitro pancreatic islet expansion medium

To identify the optimal conditions for pancreatic islet expansion in vitro, we reviewed the chemicals, proliferation and regeneration factors used in the culture of other organoids, such as those generated by pancreatic duct cells, cholangiocytes and hepatocytes ${ }^{14,16,20,21}$, and pancreatic islet organoids derived from stem cells or fibroblasts (Fig. 1) $)^{1-4}$. According to their reported functions, these chemicals and factors are classified into the following groups: islet identity, cell division and proliferation, and cell renewal and regeneration (Fig. 1). By screening different combinations of compounds and factors, a medium composed of 12 chemicals and hormones, as well as the general nutrients nicotinamide, B27 and GlutaMAX, was created and found to robustly support the in vitro expansion of dispersed islet clusters isolated from pregnant mice or wild-type rats. We named this in vitro pancreatic islet expansion medium pancreatic islet expansion medium (PIEM).

\section{Expansion of mouse islet clusters in vitro}

Previous studies have shown that pregnant mouse islets maintain expansion ability in vivo, whereas wild-type mouse islets undergo little expansion ${ }^{22,23}$. We therefore used islets from both pregnant and wild-type mice, as 
pregnant mice could serve as an easier starting point for investigating the regeneration process. We isolated pancreatic islets from both wild-type and pregnant C57BL/6 mice by collagenase $\mathrm{P}$ perfusion, sedimentation and handpicking. These pancreatic islets were separated into single cells or cell clusters by Dispase II digestion and mechanical dissociation. The single cells or cell clusters were cultured in 3-dimensional (3D) Matrigel. Only dispersed islet clusters isolated from pregnant mice showed significant expansion during nine-day culture (Fig. 2a). Approximately $5 \%-15 \%$ of the pancreatic islet clusters isolated from pregnant mice were able to expand from a surface area of $6000-9000 \mu^{2}(240-320$ cells $)$ to a surface area of $20,000-30,000 \mu \mathrm{m}^{2}$ (1300-2100 cells) (Fig. 2a, c). In contrast, the dispersed single islet cells from pregnant or wild-type mice or islet clusters from wild-type mice showed no significant expansion (Fig. 2b, d).

We next used qRT-PCR to examine the expression of key islet markers in the expanded islet clusters. The expanded islet clusters maintain expression of the crucial pancreatic $\beta$ cell markers insulin-1 (Ins1), insulin-2 (Ins2) and Mafa; the $\alpha$ cell markers glucagon (Gcg) and Mafb; and the $\delta$ cell marker somatostatin (Sst) (Fig. 2e).

To confirm that specific cell types coexisted in the in vitro-expanded islet clusters, which is a hallmark of expanded islet clusters, we performed immunostaining for insulin, glucagon and somatostatin, which are markers for pancreatic $\alpha$ cells, $\beta$ cells and $\delta$ cells, respectively. The staining result unambiguously identified that glucagon, insulin, and somatostatin signals were present in different cells grouped together in the same expanded islet clusters similar to primary islets (Fig. 3a, b; Supplementary Fig. S1a, b). The percentage of the $\alpha$ cells, $\beta$ cells and $\delta$ cells are $(12.4 \pm 3.67) \%,(78 \pm 4.55) \%, \quad(7.68 \pm 1.26) \%$, respectively, in propagated islet clusters, which is similar to the pancreatic islets isolated from pregnant mice (Fig. 3c). Importantly, we also observed the co-localisation of the pancreatic transcription factors PDX1 and MAFA with insulin, as well as the partial co-localisation of NKX6.1 with insulin in the same cells of expanded islet clusters, which is similar to the pancreatic islets isolated from pregnant mice (Fig. 3d-f; Supplementary Fig. S1c-e).

We next examined the functional integrity of the expanded islet clusters. In response to combined stimulation with $20 \mathrm{mM}$ glucose and $100 \mathrm{nM}$ glucagon peptide 1 (GLP-1), the expanded islet clusters demonstrated a significant increase in insulin secretion, although with a smaller extent compared to the pregnant mouse islets (Fig. 3g). In response to high-level glucose stimulation, both other labs and our lab have shown that UCN3 serves as an endogenous paracrine factor secreted by pancreatic $\beta$ cells to stimulate endogenous somatostatin secretion from pancreatic $\delta$ cells ${ }^{7,10,24}$. We therefore stimulated the expanded islet clusters with both glucose and UCN3 to minimise the amount required for islet cluster usage. The in vitro-expanded islet clusters displayed significantly more somatostatin release in response to glucose and UCN3 stimulation (Fig. 3h). Moreover, the expanded islet clusters secreted more glucagon in response to $25 \mathrm{mM}$ arginine stimulation, hallmarking the normal function of pancreatic islet $\alpha$ cells, which is similar to the pregnant mouse islets (Fig. 3i). These results confirmed the functional integrity of the in vitro-expanded islet clusters isolated from pregnant mice.

Although the expanded pancreatic islet clusters showed similar somatostatin and glucagon secretion compared to the primary isolated islets in response to specific physiological stimulations, it is worth noting that their insulin secretion in response to the combined stimulation of glucose and GLP-1 is significantly weakened. Some extent of degeneration during the islet expansion may occur under the current in vitro expansion condition, which awaits for further investigation and method optimisation.

\section{Expansion of rat islet clusters in vitro}

We then examined the expansion of the dispersed rat islet single cells and clusters in PIEM. Similar to those from pregnant mice, $\sim 5 \%-15 \%$ of the rat pancreatic islet clusters were able to expand from a surface area of $5000-8000 \mu^{2}(200-350$ cells) to a surface area of $20,000-25,000 \mu \mathrm{m}^{2}$ (1000-1900 cells), whereas dispersed rat islet single cells showed no such expansion ability (Fig. 4a, b). Furthermore, qRT-PCR revealed that the expanded rat islet clusters maintained the same expression levels of the $\alpha$ cell markers Gcg and Mafb as the primary rat islets, but showed significantly higher expression of the $\beta$ cell markers Ins-1, Ins-2 and Mafa and the $\delta$ cell marker Sst (Fig. 4c).

\section{Increased gene expression related to dedifferentiation, pluripotency and proliferation in expanded islet clusters}

We next examined whether the expanded islet clusters increased the expression levels of genes functionally associated with cell proliferation, renewal and regeneration, which are important factors for in vitro regeneration. Importantly, significantly higher expression of Ki67, Ccnd 1 and Pcna was found in expanded islet clusters than in islets derived from pregnant mice or wild-type rats, highlighting the increased proliferation ability of the expanded islet clusters (Fig. 5a, d). Moreover, significantly higher levels of Nanog and Sox9 expression were found in expanded islet clusters derived from both pregnant mice and rats (Fig. 5b, e). These results provide putative explanations accounting for the better proliferation and renewal abilities of isolated islet clusters than integral islets.

We suspected that these islets gained proliferation capacity and pluripotency due to dedifferentiation. Thus, we examined dedifferentiation markers in expanded islet 


\section{a}
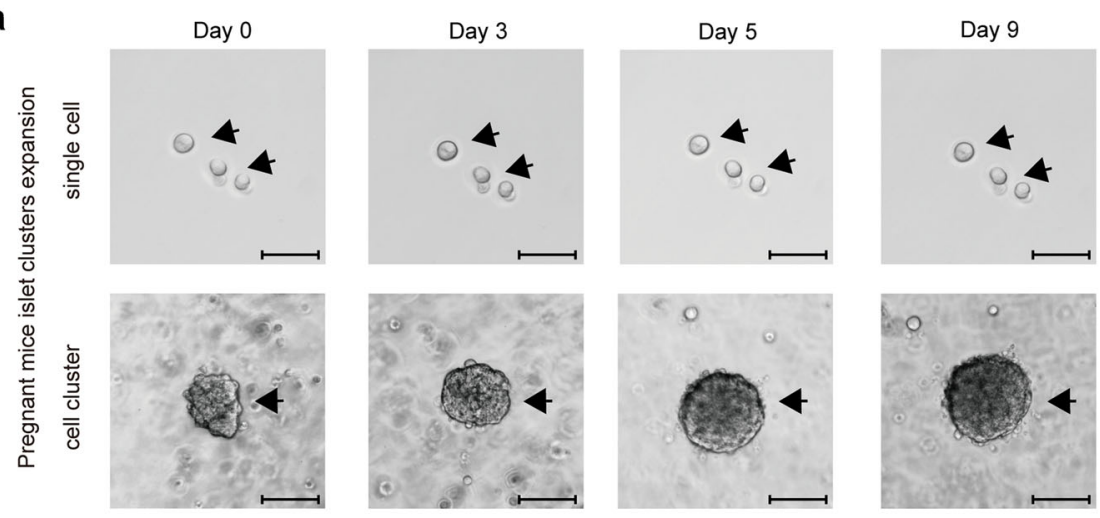

b

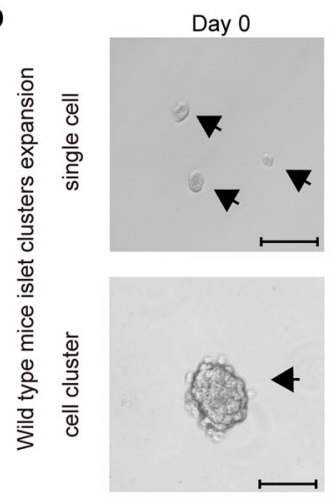

Day 3

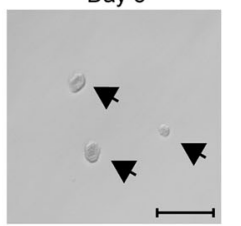

Day 5

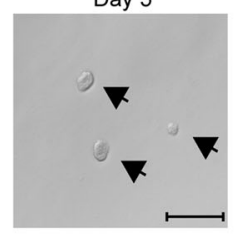

Day 9

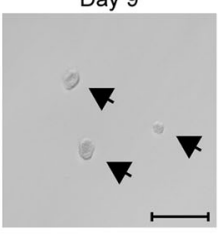

C
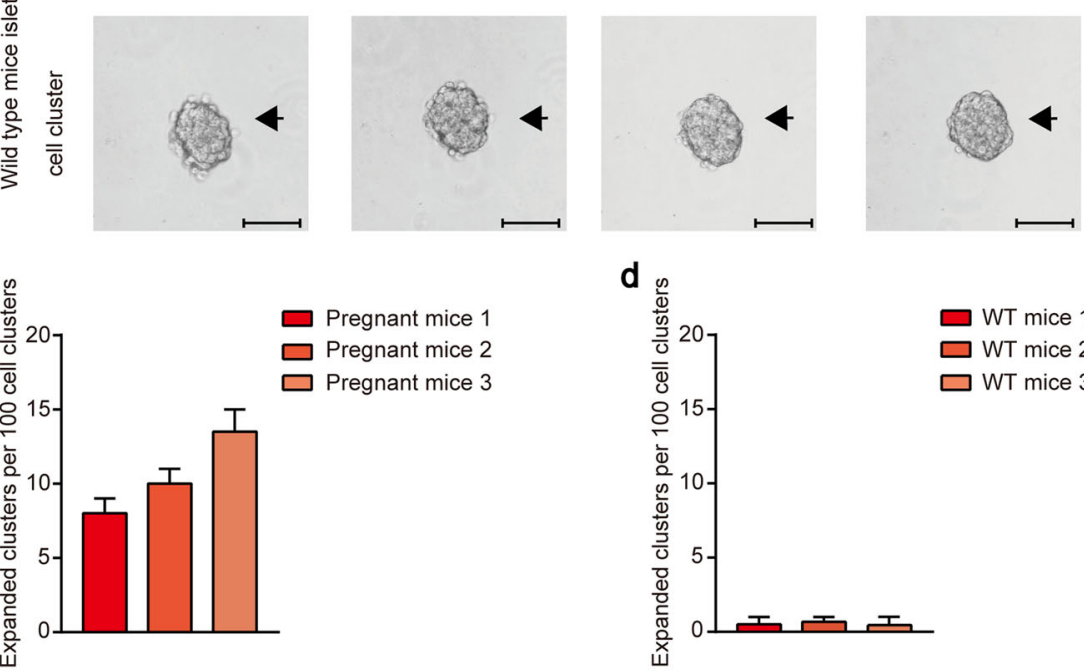

d

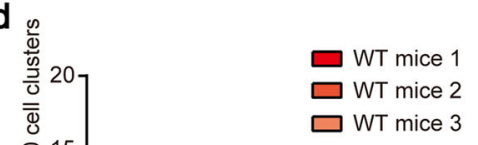

e

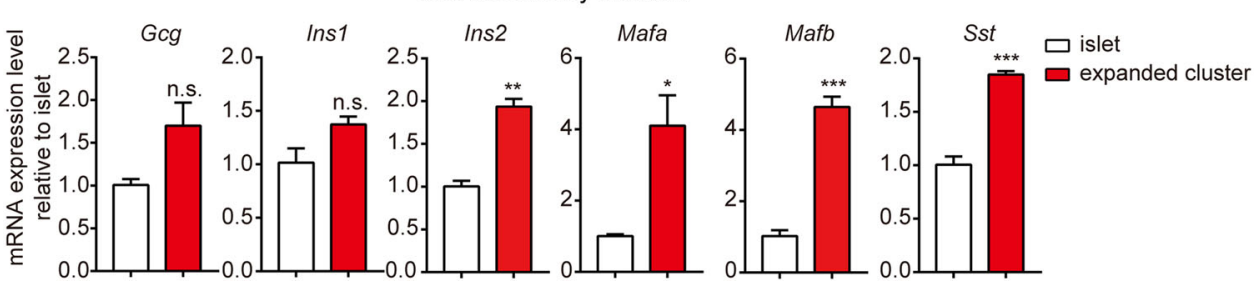

Fig. 2 In vitro expansion of pregnant mouse islet clusters. a Representative images of single islet cells or islet clusters $\left(\sim 6000-9000 \mu m^{2}\right.$ original surface area) derived from pregnant mice after 3D culture in pancreatic islet cluster expansion medium (PIEM) at the indicated time points. Experiments were performed on pregnant mice. The scale bar in the single-cell image represents $50 \mu \mathrm{m}$, and one in the cluster image represents $100 \mu \mathrm{m}$. b Representative images of dispersed wild-type mouse islet single cells or clusters at various times after 3D culture with PIEM. The scale bar in the single-cell image represents $50 \mu \mathrm{m}$, and one in the cluster image represents $100 \mu \mathrm{m}$. c Numbers of expanded clusters per 100 islet cell clusters. The experiments were performed in triplicate and with pregnant mice. The data are represented as the mean \pm SEM. $\mathbf{d}$ Numbers of expanded clusters per 100 islet cell clusters. The experiments were performed in triplicate on wild-type mice. The data are represented as the mean \pm SEM. e Comparison of islet cell identity marker expression in pregnant mouse pancreatic islet clusters vs isolated primary mouse islets. ${ }^{*} P<0.05$; ${ }^{* *} P<0.01$; ${ }^{* * *} P<0.001$; clusters were compared with primary mouse islets. The data are shown as the mean \pm SEM of at least three independent experiments. Data were analysed using unpaired two-tailed Student's $t$-test. 


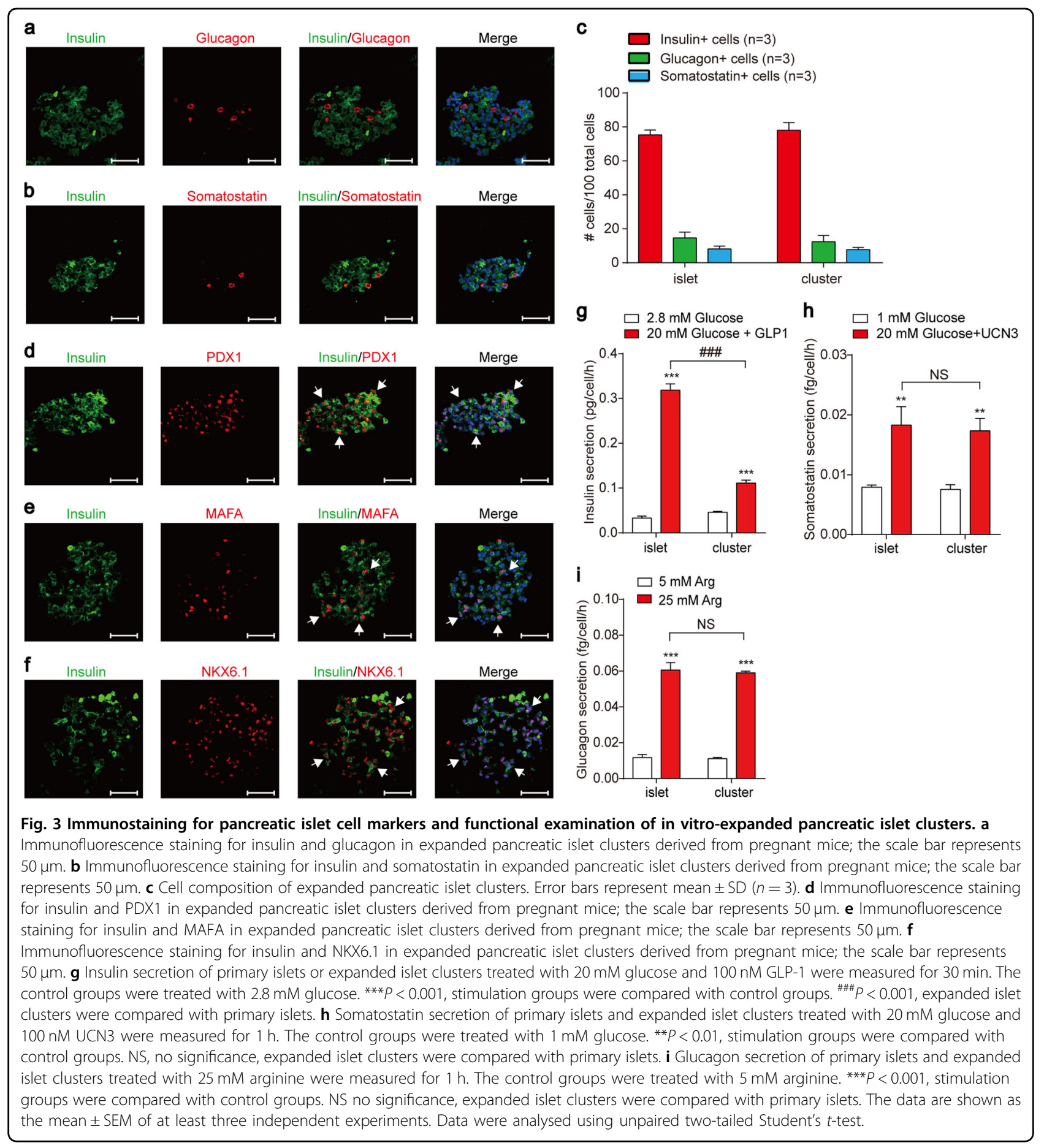

clusters and compared them with those in isolated pancreatic islets. Interestingly, the expanded islet clusters showed significantly higher expression of Ngn3 and $H l x b 9$, markers that characterise pancreatic islet progenitor cells (Fig. 5c). Although the expanded islet clusters derived from pregnant mice showed higher expression of Gata6, they showed decreased expression of Gata4, both of which are pancreatic progenitor markers
(Fig. 5c, f). The protein expression of KI67 and SOX9 in expanded islet clusters were higher than that in primary islets, as confirmed by immunofluorescence (Supplementary Fig. S2a-d). Similarly, the expanded islet clusters derived from rats did not display significant pancreatic progenitor characteristics, as they showed decreased expression of Gata6 (Fig. 5c, f). The data suggested that these expanded islet clusters underwent one-step 

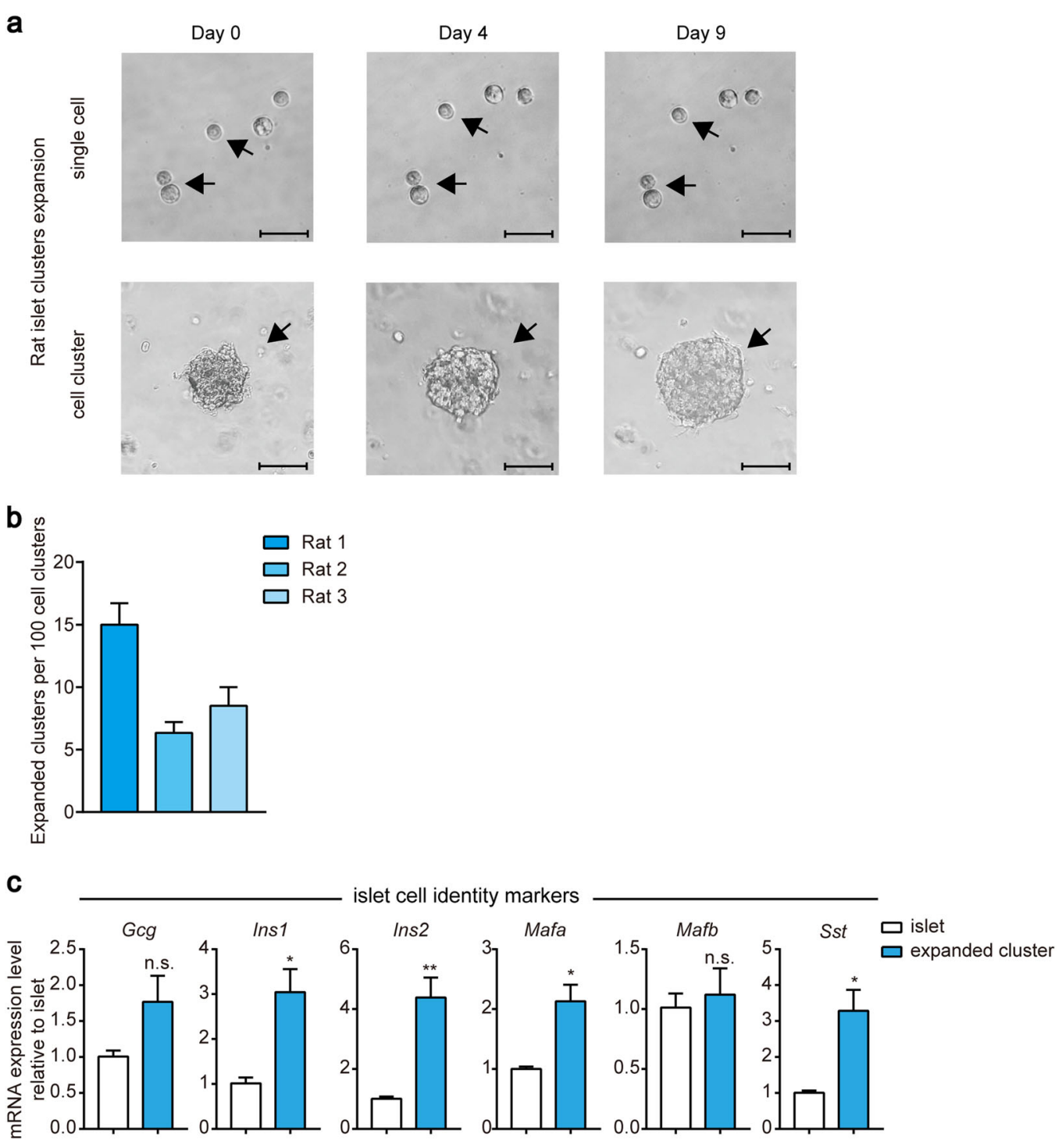

Fig. 4 In vitro expansion of rat islet clusters. a Representative images of rat islet single cells or clusters ( 5000-8000 $\mu \mathrm{m}^{2}$ original surface area) at various time points after 3D culture with PIEM. The scale bar in the single-cell image represents $50 \mu \mathrm{m}$, and one in the cluster image represents $82.5 \mu \mathrm{m}$. b Numbers of expanded clusters per 100 islet cell clusters. The experiments were performed on rats in triplicate. The data are shown as the mean \pm SEM. c Comparison of the expression of islet cell identity markers in rat-expanded clusters compared with primary islets. ${ }^{*} P<0.05$; ${ }^{* *} P<0.01$; clusters were compared with primary rat islets. The data are shown as the mean \pm SEM of at least three independent experiments. Data were analysed using unpaired two-tailed Student's t-test.

dedifferentiation towards islet progenitor cells, but they did not ultimately reach the pancreatic progenitor cell stage. Taken together, this one-step dedifferentiation of isolated islet clusters cultured in PIEM towards pancreatic islet progenitor cells contributed to the gain of cell proliferation, renewal and regeneration abilities of expanded islet clusters.

\section{Essential roles of FSK and exendin-4 in PIEM}

During our formula component screening and recipe formation for PIEM, we identified that FSK and exendin-4 are both required for robust islet cluster expansion in vitro. It is known that exendin-4 induced cAMP accumulation after it activates GLP-1R and the downstream Gs proteins ${ }^{25}$. FSK is known as a cAMP agonist through direct binding to adenyl cyclase ${ }^{26}$. Moreover, recent reports indicate that FSK is required for proliferation of liver organoids in addition to A83- $01^{27}$. Interestingly, removing FSK or exendin-4 from PIEM had different effects on the expression profiles of marker genes related to islet identity, proliferation, renewal and regeneration. Removing FSK from PIEM had partial 


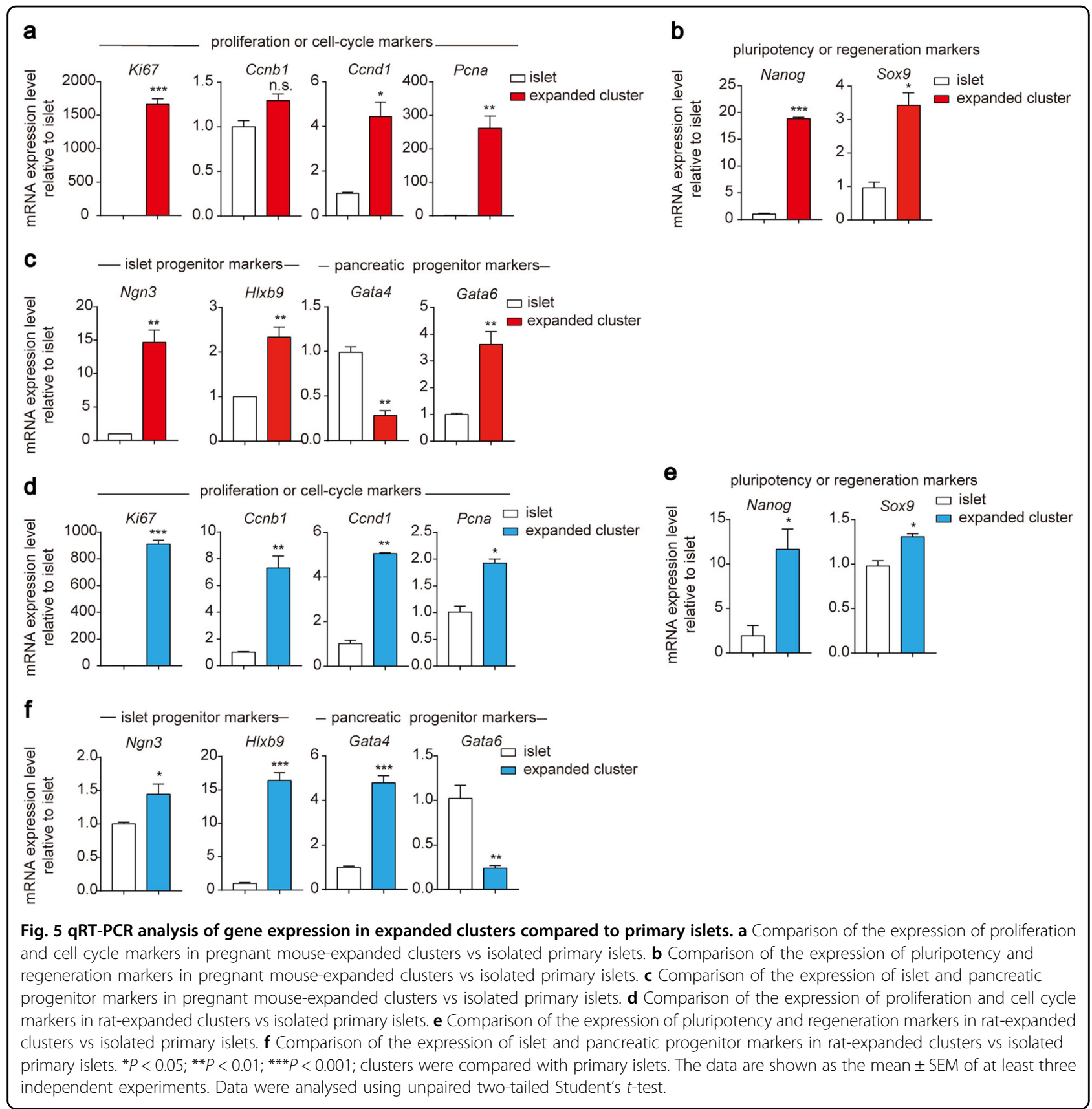

effects on islet identity and cell proliferation markers, including decreased expression of Mafa, Sst and Ccnb1 (Fig. 6a, b). In particular, FSK was required for the expression of cell renewal and regeneration markers, including Nanog and Sox17 (Fig. 6c). In contrast, exendin4 was essential for islet identity and proliferation because it maintained the expression of the pancreatic $\beta$ cell markers Ins-1, PdxI and Mafa and the proliferation markers Ki67, Ccnb1 and Cdk4 (Fig. 6a, b). In particular, exendin-4 was required for the expression of $\mathrm{Ngn} 3$ and $H x l b 9$, two islet progenitor markers (Fig. 6d). These results indicated that FSK in PIEM renders renewal and regeneration ability to isolated islet clusters, whereas exendin- 4 is essential for maintaining islet $\beta$ cell identity, proliferation and facilitating dedifferentiation.

\section{Discussion}

The in vitro expansion of primary pancreatic $\beta$ cells or pancreatic islet tissues has not been reported before. Here, we developed a medium recipe, which we named PIEM, that enabled the proliferation of dispersed islet clusters isolated from pregnant mice or wild-type rats in vitro 

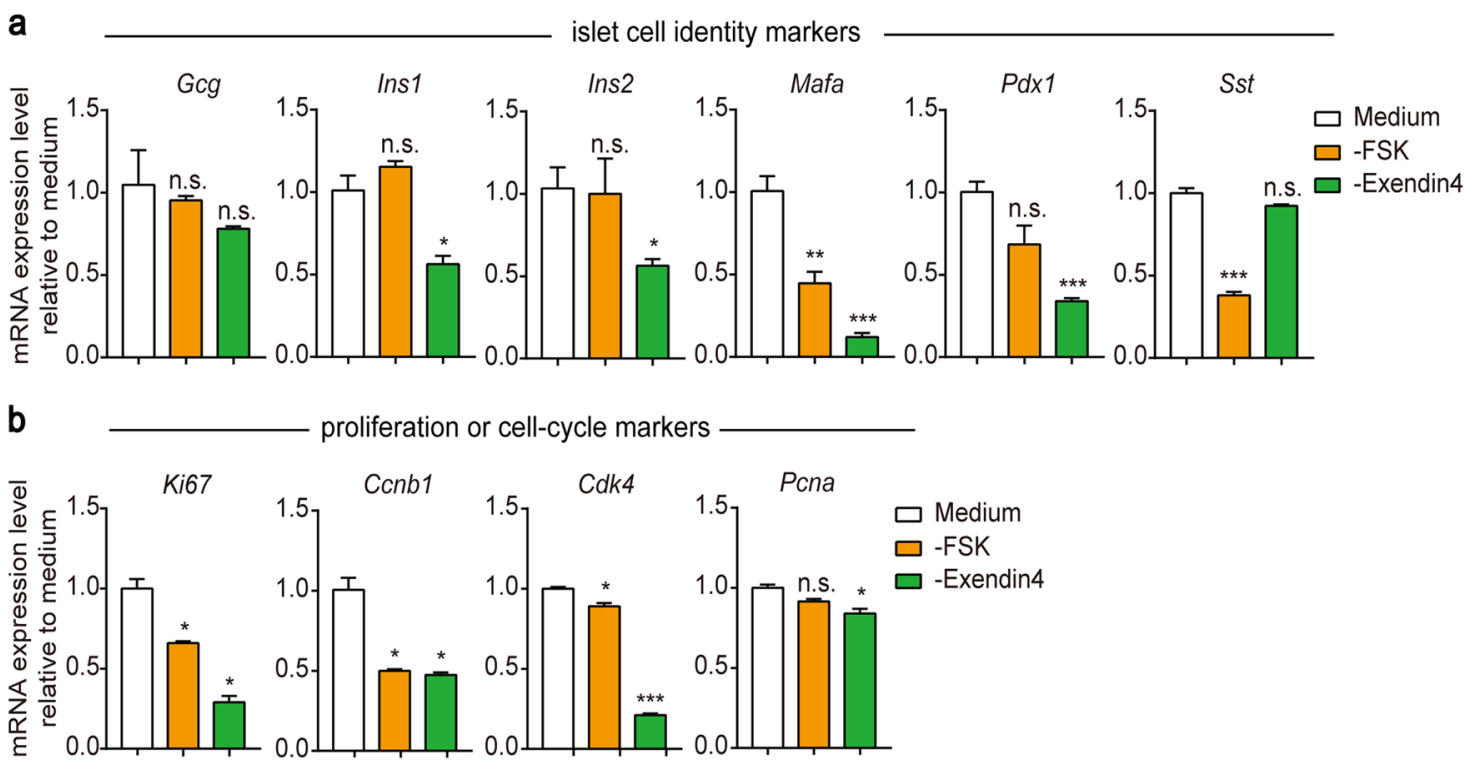

C pluripotency or regeneration markers
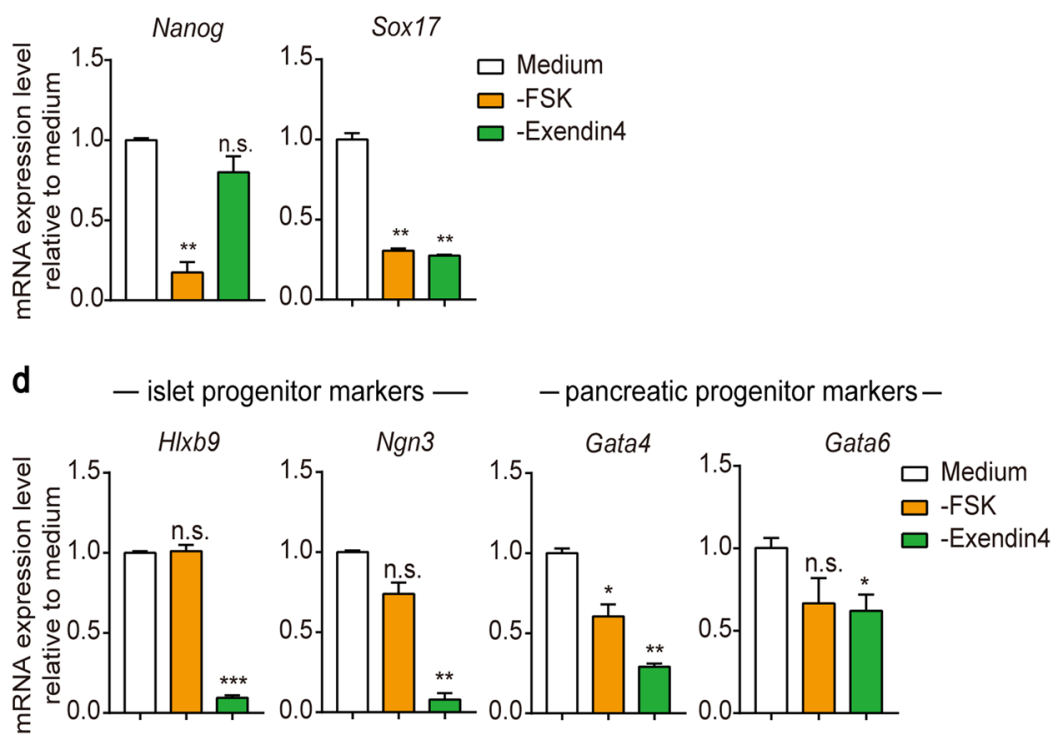

Fig. 6 Essential roles of FSK and exendin-4 in in vitro pancreatic islet expansion. a-d qRT-PCR analysis of the expression of islet cell identity markers (a), proliferation and cell cycle markers (b), pluripotency and regeneration markers (c) and islet and pancreatic progenitor markers (d) in pregnant mouse-expanded clusters after 3 days of culture with PIEM or PIEM without FSK or exendin-4. ${ }^{*} P<0.05$; ${ }^{* *} P<0.01$; ${ }^{* *} P<0.001$; cell clusters cultured in PIEM without FSK or exendin-4 were compared with those cultured in complete PIEM. The data are shown as the mean \pm SEM of at least three independent experiments. Data were analysed using unpaired two-tailed Student's t-test.

(Fig. 7). Isolated islet clusters normally grow from an initial surface area of $5000-9000 \mu \mathrm{m}^{2}$ to a surface area of 20,000-30,000 $\mu \mathrm{m}^{2}$, with an estimated 5-8-fold increase in cell number. Compared to primary pancreatic islets, expanded cell clusters have similar or higher mRNA expression levels of insulin, glucagon and somatostatin, which are markers of pancreatic $\beta$ cells, $\alpha$ cells and $\delta$ cells, respectively. Expanded islet clusters demonstrated normal insulin and somatostatin secretion in response to physiological stimulation and normal glucagon secretion in response to amino acids, suggesting that the clusters recapitulate specific functions of pancreatic islets. Immunostaining further confirmed that the cells expressing insulin, glucagon and somatostatin were grouped together and spatially organised in expanded islet clusters similar to primary islets. These data indicated that the expanded islet clusters behave similar to an in vitro organoid, thus serving as an initial step for future 


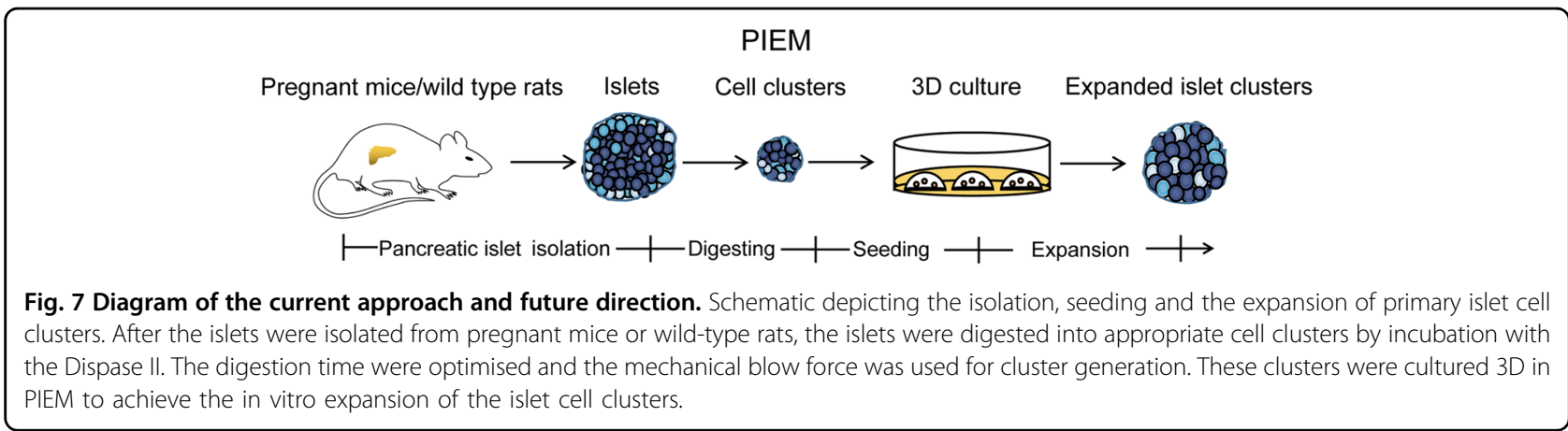

development of in vitro islet propagation methods. The ultimate goal of developing efficient methods for propagation islets in vitro is to fulfil the demand for regenerative diabetes therapy and personalised drug tests.

It is worth noting that we observed the expansion of islet clusters isolated from only pregnant mice and wildtype rats in PIEM, not those isolated from wild-type mice. Pregnant mice are well known for their increased pancreatic $\beta$ cell proliferation ${ }^{28-31}$, which provides a useful starting point for testing medium recipes. Although we did not observe the proliferation of islet clusters isolated from wild-type mice, we did observe the expansion of islet clusters isolated from wild-type rats, which share more properties identical to human islets than mouse islets. An urgent need is to test whether PIEM enables the expansion of primary islet clusters isolated from human patients.

Another notable observation is that only dispersed islet clusters were able to proliferate in vitro, whereas dispersed single primary islet cells could not. Two important factors may contribute to this discrepancy. First, the islet clusters contain multiple cell types, which not only are the origins of the different cell types in the finally expanded islet clusters but also may form certain cell circuits and gradient hormone concentrations to support in vitro expansion. Second, primary single cells must undergo harsher digestion than islet clusters, which means that the extracellular parts of the membrane or matrix proteins, including receptors, ion channels and transporters, may be digested with their functions impaired. These membrane or matrix proteins may be key factors for in vitro tissue expansion. Therefore, future analysis of the different expression patterns and functions of membrane proteins and signalling circuit differences between isolated islet clusters and single cells may provide more clues for unravelling the secrets of the in vitro expansion of islets and provide guidance for a better strategy for in vitro islet cluster expansion.

We noticed that expanded islet clusters have higher expression of $N g n 3$ and $H l x b 9$, which are markers of pancreatic islet progenitor cells. Although the expression level of Nanog in islet clusters is $\sim 10$ times as much as that in normal islets, this level is closer to that in the whole embryo tissue but much lower (more than 1000 fold lower) than that in the embryo stem cells (Supplementary Fig. S2e). Whether this amount of Nanog is required for islet expansion and propagation in vitro, and whether it has cancer potential require further investigation. This observation indicated that in vitro-expanded islets underwent certain dedifferentiation. However, we doubt that the islet clusters gained proliferation ability through this dedifferentiation. Further experiments with withdrawal of key chemicals in the medium will test this hypothesis. For clinical usage, the in vitro expansion of 100 patient islets to 1 million islets without significant gene mutations, while preserving pancreatic islet function, and transferring them back to the patient to recover glucose homoeostasis is ideal. However, the current approach of PIEM allows only 5-8-fold growth of $\sim 5 \%-15 \%$ of isolated islet clusters. The expansion of the islet clusters was only observed in the first passage. Therefore, it is unlikely to cause significant genomic instability. However, the amount of the currently acquired in vitro-expanded islet cluster cells is not sufficient for RNA-seq to verify the gene expression profile or transplantation to confirm their in vivo functions. More importantly, the final usage of in vitro-propagated islet clusters for regenerative therapy to treat diabetes in general requires millions of pancreatic islets. Therefore, a future revision of the current PIEM composition and the method for in vitro islet propagation is in an urgent demand.

One of the future directions for the optimisation of in vitro islet propagation methods could be as follows: (1) initial expansion and dedifferentiation of islet clusters to islet progenitor-like cell clusters, (2) rapid proliferation of islet progenitor-like cell clusters to a sufficient amount, (3) differentiation of progenitor-like cell clusters to functional integral islets, (4) transplantation or (5) exploitation of these expanded islet tissues for drug tests. Therefore, manifesting the markers for each stage and searching for chemicals that are useful for each procedure 
in the above protocol is our next goal to fulfil the regeneration of the expanded islet clusters in vitro in the future.

Taken together, we developed a recipe for PIEM in this study, and our 3D culture method enabled the initial generation of expanded islet clusters via in vitro expansion of the isolated islet clusters from pregnant mice or wild-type rats in the first passage. Our study is one important step towards the realisation of a method to fulfil in vitro propagation of pancreatic islets, which may facilitate the development of new therapies to treat diabetes.

\section{Materials and methods \\ Animals}

C57BL/6 mice and rats of Wistar strain were housed and bred under specific-pathogen-free conditions at Shandong University animals care facility. Female animals were used. The numbers of animals are indicated in the figure legends within each experiment. All animal care and experiments were reviewed and approved by the Animal Use Committee of Shandong University School of Medicine.

\section{Pancreatic islet isolation and digestion into single cells or cell clusters}

We isolated pancreatic islets from C57BL/6 mice, pregnant C57BL/6 mice and Wistar rats. Briefly, mice and rats were killed by cervical dislocation and then pancreata were isolated from them individually. Adult pancreata were digested by Collagenase P (Roche, 11213873001) at $37^{\circ} \mathrm{C}$ for $18-25 \mathrm{~min}$. Digestion was stopped by cold Hank's balanced salt solution $(136.9 \mathrm{mM} \mathrm{NaCl} ; 5.4 \mathrm{mM}$ $\mathrm{KCl} ; 1.3 \mathrm{mM} \mathrm{CaCl} ; 0.8 \mathrm{mM} \mathrm{MgSO}$; $0.44 \mathrm{mM} \mathrm{KH}_{2} \mathrm{PO}_{4}$; $0.34 \mathrm{mM} \quad \mathrm{Na}_{2} \mathrm{HPO}_{4} ; \quad 5.55 \mathrm{mM} \quad$ D-glucose; $4.4 \mathrm{mM}$ $\mathrm{NaHCO}_{3}, \mathrm{pH}$ 7.4) followed by sedimentation for three or five times at $4{ }^{\circ} \mathrm{C}$. The islets were collected by hand picking using a stereomicroscope and were cultured overnight in islet complete media containing $5.6 \mathrm{mM}$ glucose (Biological Industries), 10\% FBS (Gibco), 0.1\% penicillin/streptomycin. Next we obtained single cells and cell clusters of different diameters $(20-150 \mu \mathrm{m})$ under the microscope by controlling enzyme concentration, digestion and settling time. Islets were naturally settled for $1 \mathrm{~min}$ in KRBB buffer $(135 \mathrm{mM} \mathrm{NaCl} ; 4.7 \mathrm{mM} \mathrm{KCl}$; $1.2 \mathrm{mM} \mathrm{KH}{ }_{2} \mathrm{PO}_{4} ; 10 \mathrm{mM}$ Hepes; $3 \mathrm{mM}$ D-glucose; $5 \mathrm{mM}$ $\mathrm{NaHCO}_{3} ; 0.1 \%$ BSA; $1 \%$ penicillin/streptomycin, pH 7.4), preheated EDTA-KRBB solution was added, gently piped for $2 \mathrm{~min}$, then placed in a $33^{\circ} \mathrm{C}$ water bath for $10 \mathrm{~min}$; after removing the supernatant, tissues were digested with Dispase II (0.1-0.5 U/ml, Roche, 04942078001$)$ in a $33^{\circ} \mathrm{C}$ water bath for 3-8 min to obtain single cells or islet cell clusters. Mechanical fragmentation was achieved by gentle pipetting (5-10 times) with a glass dropper in a $15 \mathrm{~mL}$ glass centrifuge tube. Using DMEM/F-12 (Biological Industries) medium with 10\% FBS (Gibco) and 1\% penicillin/streptomycin for termination of digestion. Collecting single cells or cell clusters by centrifugation ( $300 \times g, 5 \mathrm{~min}$, room temperature).

\section{Production of RSPO1-conditioned medium}

Plasmid encoding mRSPO1 was a gift from Dr. Ren-Jie Chai (Southeast University, Nanjing, CN). HEK293T cells were passaged and transfected with plasmid encoding mRSPO1. The medium containing secreted RSPO1 protein was collected and verified by western blotting. The supernatant was filtered through $0.45 \mu \mathrm{m}$ filters and used for further applications ${ }^{32}$.

\section{Culture of expanded islet clusters}

Isolated islet cell clusters were washed twice with DMEM/F-12 (Biological Industries), counted and mixed with Matrigel in glass centrifuge tubes. In all, $30,000-50,000$ cells or $10-20$ cell clusters were used per well of a pre-warmed 24-well plate (the volume of $50 \mu \mathrm{L}$ ). After Matrigel was solidified for $30 \mathrm{~min}$ at $37^{\circ} \mathrm{C}$, pancreatic islet expansion medium (PIEM) was added. PIEM consists of DMEM/F-12 (1\% GlutaMax, 1\% PenicillinStreptomycin) plus 15\% RSPO1 conditioned medium (home-made), $3 \mu \mathrm{M}$ CHIR-99021 (BioGems), $1 \mu \mathrm{M}$ A8301 (Adooq Bioscience), $10 \mathrm{nM}$ gastrin-1 (MedChemExpress), $1.25 \mathrm{mM} \mathrm{N}$-acetylcysteine (Sigma), $10 \mu \mathrm{M} \mathrm{Y-27632}$ (Adooq Bioscience), $10 \mu \mathrm{M}$ FSK (TargetMol), $50 \mathrm{ng} / \mathrm{mL}$ Exendin-4 (ChinaPeptides), $50 \mu \mathrm{g} / \mathrm{mL}$ L-Ascorbic acid (Sigma), $250 \mathrm{nM}$ 5-Iodotubercidin (Adooq Bioscience), 50 ng/mL FGF10 (PeproTech), 50 ng/mL EGF (PeproTech), $10 \mathrm{mM}$ Nicotinamide (Sigma), B27 Supplement (minus Vitamin A) (Thermo). Cultures were kept at $37^{\circ} \mathrm{C}$, $5 \% \mathrm{CO}_{2}$ in a humidified incubator. During culturing, medium was refreshed every three days.

\section{RNA extraction and qRT-PCR}

RNA from islets and islet single cells as well as islet cell clusters derived from C57BL/6 mice, pregnant C57BL/6 mice and Wistar rats, including the corresponding islet clusters were extracted with TRIzol reagent (Thermo). We used the tip to pick up the expanded islet clusters from the Matrigel using a stereomicroscope. Cell recovery solution (Corning) is used to recover cells from Matrigel. Cold cell recovery solution $(0.2-0.5 \mathrm{~mL}$ per well) is added. Scrape the gel layer into a cold $1.5 \mathrm{~mL}$ microcentrifuge tube and leave it on ice for $0.5-1 \mathrm{~h}$ until the gel completely dissolved. Cells were collected by centrifugation $\left(200 \times g, 5 \mathrm{~min}, 4^{\circ} \mathrm{C}\right)$. And we used TURBO DNA-free Kit (Invitrogen) to remove DNA contamination from the extracted RNA. cDNA was synthesised using the 
qRT-PCR Kit (Toyobo, FSQ-101). qRT-PCR was conducted in the LightCyclerqPCR apparatus (Bio-Rad) with the FastStart SYBR Green Master (Roche). All primers used for qRT-PCR assay are listed in Supplementary Table S1.

\section{Immunofluorescence staining}

Expanded islet clusters were harvested using cell recovery solution (Corning, 354253) and fixed in 4\% paraformaldehyde for $30 \mathrm{~min}$ at room temperature, followed with blocking and permeabilizing in PBS with $0.5 \%$ Triton X-100 (Solarbio, T8200) and 5\% donkey serum (Solarbio, SL050) for $30 \mathrm{~min}$ at room temperature. Then, samples were incubated with primary antibody at $4{ }^{\circ} \mathrm{C}$ overnight, followed by incubation with secondary antibody for $2 \mathrm{~h}$ at room temperature. DAPI (Beyotime, C1002) was used to stain the nucleus and find islets. The following antibodies were used for immunofluorescence: anti-insulin (1:200, sc-9168; Santa), anti-somatostatin (1:600, ab30788; Abcam), antiglucagon (1:200, G2654; Sigma). anti-PDX1 (1:200, ab47267; abcam), anti-SOX9 (1:200, ab185966; abcam), anti-NKX6.1 (1:200, ab221549; abcam), anti-MAFA (1:200, ab26405; abcam), anti-KI67 (1:200, D3B5; Cell Signaling Technology). Imaging of the expanded islet clusters was performed on Zeiss LSM 780 and processed using ImageJ or Adobe illustrator software.

\section{Insulin secretion measurement}

The insulin secretion analysis was preformed similarly to previously described by our group ${ }^{7,9,24}$. Fifty day 7 expanded islet clusters (cell number is comparable to $1 / 10$ of primarily isolated islets, $1000-2000$ cells) or 10 islets for each group were kept in $2.8 \mathrm{mM}$ glucose MKRBB buffer $(5 \mathrm{mM} \mathrm{KCl}, 120 \mathrm{mM} \mathrm{NaCl}, 15 \mathrm{mM}$ Hepes, $24 \mathrm{mM}$ $\mathrm{NaHCO}_{3}, 1 \mathrm{mM} \mathrm{MgCl}, 2 \mathrm{mM} \mathrm{CaCl} 2,2.8 \mathrm{mM}$ Glucose, $\mathrm{pH}=7.4)$ for $1 \mathrm{~h}$ at $37^{\circ} \mathrm{C}$, and then treated with $20 \mathrm{mM}$ glucose and $100 \mathrm{nM}$ GLP1, whereas the control groups were treated with $2.8 \mathrm{mM}$ glucose for $30 \mathrm{~min}$. The supernatant fractions were collected to measure the secreted insulin. The insulin levels were measured with the Millipore Rat/Mouse Insulin ELISA kit(Cat. \# EZRMI$13 \mathrm{~K})$ as per the manufacturer's instructions.

\section{Somatostatin measurement}

The somatostatin secretion analysis was preformed similarly to previously described by our group ${ }^{7,9,24}$. Fifty day 7 expanded islet clusters or 10 primary islets for each group were kept in $1 \mathrm{mM}$ glucose MKRBB buffer for $1 \mathrm{~h}$ at $37^{\circ} \mathrm{C}$, then treated with $20 \mathrm{mM}$ glucose and $100 \mathrm{nM}$ UCN3, whereas the control groups were treated with $1 \mathrm{mM}$ glucose for $1 \mathrm{~h}$. The supernatant fractions were collected for somatostatin measurement using the Phoenix Pharmaceuticals ELISA kit (EK-060-03) as per the manufacturer's instructions.

\section{Glucagon measurement}

The glucagon secretion analysis was preformed similarly to previously described by our group ${ }^{7,9,24}$. Fifty day 7 expanded islet clusters or ten primary islets for each group were kept in $12 \mathrm{mM}$ glucose MKRBB buffer for $1 \mathrm{~h}$ at $37^{\circ} \mathrm{C}$, and then treated with $1 \mathrm{mM}$ glucose and $25 \mathrm{mM}$ arginine, whereas the control groups were treated with $5 \mathrm{mM}$ Arginine for $1 \mathrm{~h}$. The supernatant fractions were collected to measure the secreted glucagon. The glucagon levels were measured with the Cloud-Clone Corp Mouse Glucagon ELISA kit (Cat. CEB266Mu) as per the manufacturer's instructions.

\section{Statistical analysis}

qRT-PCR was performed independently for at least three times. Statistical analyses were performed using GraphPad Prism 7 software. Experimental data were analysed using unpaired two-tailed Student's $t$-test. Results were presented as mean \pm SEM; $P<0.05$ was considered statistically significant.

\section{Acknowledgements \\ This work was supported by funding from the National Key Basic Research Program of China Grant (2018YFC1003600 to J.-P.S. and X.Y., 2019 YFA0111400 to $H$-.L.H.), the National Science Fund for Excellent Young Scholars Grant (81822008 to X.Y.), the National Science Fund for Distinguished Young Scholars Grants (81825022 to J.-P.S.), the National Natural Science Foundation of China (31671197 to X.Y., 81773704 to J.-P.S., 31970779 to H.-L.H., and 81173086 to J. A.), the Distinguished Young Scholars Grants of Shandong Province (JQ201517 to J.P.S.) and the Shandong Key Research and Development Program (2017GSF18138 to J.A.).}

\section{Author details \\ ${ }^{1}$ Key Laboratory Experimental Teratology of the Ministry of Education and Department of Physiology and Pathophysiology, School of Basic Medical Sciences, Shandong University, 250012 Jinan, Shandong, China. ${ }^{2}$ Department of Physiology and Pathophysiology, School of Basic Medical Sciences, Peking University, Key Laboratory of Molecular Cardiovascular Science, Ministry of Education, 100191 Beijing, China. ${ }^{3}$ Key Laboratory for Developmental Genes and Human Disease of the Ministry of Education, School of Life Science and Technology, Southeast University, 210096 Nanjing, Jiangsu, China. \\ ${ }^{4}$ Department of Pharmacology, School of Basic Medical Sciences, Shandong University, 250012 Jinan, Shandong, China. ${ }^{5}$ Key Laboratory Experimental Teratology of the Ministry of Education and Department of Biochemistry and Molecular Biology, Shandong University School of Medicine, 250012 Jinan, Shandong, China. ${ }^{6}$ Key Laboratory Experimental Teratology of the Ministry of Education and Department of Biochemistry and Molecular Biology, School of Basic Medical Sciences, Shandong University, 250012 Jinan, Shandong, China. ${ }^{7}$ The Key Laboratory of Experimental Teratology, Ministry of Education and Department of Genetics, School of Basic Medical Sciences, Shandong University, 250012 Jinan, Shandong, China}

\section{Author contributions}

X.Y. started the idea for in vitro expansion of islet tissues since 2010. X.Y. and J.-P.S. supervised the overall project design and execution. H.-L.H. provided key experience and protocols for in vitro 3D tissue culture and advices on initial components. X.Y., J.-P.S. and H.-L.H. initiated the project. J.-P.S. organised the screening idea. X.Y., J.P.S. and J.-Y.L. designed the screening details for chemicals and other factors. X.Y., J.-P.S., J.-Y.L. and J.C. designed the cell culture and all other experiments. J.-Y.L., J.C., Y.-Q.D., W.P., X.Y. and J.-P.S. participated in data analysis and interpretation. Z.Z., Y.-Q.D. and R.-J.C. provided the new material preparation methods. Jin.W., Z.Y., F.Y. and J.A. provided insightful ideas. Y.-F.X., H.L., W.-T.A., X.-Y.S. and Jia.W. participated in molecular biology 
and animal experiments. X.Y. and J.-P.S. wrote the paper. H.-L.H., R.-J.C., J.-Y.L., J.C., Y.-Q.D. and W.P. revised the paper and useful ideas in discussion.

\section{Conflict of interest}

The authors declare that they have no conflict of interest.

\section{Publisher's note}

Springer Nature remains neutral with regard to jurisdictional claims in published maps and institutional affiliations.

Supplementary Information accompanies the paper at (https://doi.org/ 10.1038/s41421-020-0159-x).

Received: 16 November 2019 Accepted: 8 March 2020

Published online: 07 April 2020

\section{References}

1. Zhu, S. et al. Human pancreatic beta-like cells converted from fibroblasts. Nat. Commun. 7, 10080 (2016).

2. Li, K. et al. Small molecules facilitate the reprogramming of mouse fibroblasts into pancreatic lineages. Cell Stem Cell 14, 228-236 (2014).

3. D'Amour, K. A. et al. Production of pancreatic hormone-expressing endocrine cells from human embryonic stem cells. Nat. Biotechnol. 24, 1392-1401 (2006).

4. Jiang, W. et al. In vitro derivation of functional insulin-producing cells from human embryonic stem cells. Cell Res. 17, 333-344 (2007).

5. Lancaster, M. A. \& Knoblich, J. A. Organogenesis in a dish: modeling development and disease using organoid technologies. Science 345, 1247125 (2014).

6. Clevers, H. Modeling development and disease with organoids. Cell 165 1586-1597 (2016).

7. Li, Q. et al. A cullin 4B-RING E3 ligase complex fine-tunes pancreatic delta cell paracrine interactions. J. Clin. Invest. 127, 2631-2646 (2017).

8. Dou, $H$. et al. Calcium influx activates adenylyl cyclase 8 for sustained insulin secretion in rat pancreatic beta cells. Diabetologia 58, 324-333 (2015).

9. Wang, H. M. et al. A stress response pathway in mice upregulates somatostatin level and transcription in pancreatic delta cells through Gs and betaarrestin 1. Diabetologia 57, 1899-1910 (2014).

10. van der Meulen, T. et al. Urocortin3 mediates somatostatin-dependent negative feedback control of insulin secretion. Nat. Med. 21, 769-776 (2015).

11. Sato, T. et al. Long-term expansion of epithelial organoids from human colon, adenoma, adenocarcinoma, and Barrett's epithelium. Gastroenterology 141, 1762-1772 (2011)

12. Boj, S. F. et al. Organoid models of human and mouse ductal pancreatic cancer. Cell 160, 324-338 (2015).
13. Bartfeld, S. et al. In vitro expansion of human gastric epithelial stem cells and their responses to bacterial infection. Gastroenterology 148, 126-136.e126 (2015).

14. Broutier, L. et al. Culture and establishment of self-renewing human and mouse adult liver and pancreas 3D organoids and their genetic manipulation. Nat. Protoc. 11, 1724-1743 (2016).

15. Sampaziotis, F. et al. Reconstruction of the mouse extrahepatic biliary tree using primary human extrahepatic cholangiocyte organoids. Nat. Med. 23 954-963 (2017)

16. $\mathrm{Hu}, \mathrm{H}$. et al. Long-term expansion of functional mouse and human hepatocytes as 3D organoids. Cell 175, 1591-1606.e1519 (2018).

17. Sachs, N. et al. Long-term expanding human airway organoids for disease modeling. EMBO J. 38, https://doi.org/10.15252/embj.2018100300 (2019).

18. Schutgens, F. et al. Tubuloids derived from human adult kidney and urine for personalized disease modeling. Nat. Biotechnol. 37, 303-313 (2019).

19. Xiang, C. et al. Long-term functional maintenance of primary human hepatocytes in vitro. Science 364, 399-402 (2019).

20. Huch, M. et al. Unlimited in vitro expansion of adult bi-potent pancreas progenitors through the Lgr5/R-spondin axis. EMBO J. 32, 2708-2721 (2013).

21. Hou, P. et al. Pluripotent stem cells induced from mouse somatic cells by small-molecule compounds. Science 341, 651-654 (2013).

22. Sorenson, R. L. \& Brelje, T. C. Adaptation of islets of Langerhans to pregnancy: beta-cell growth, enhanced insulin secretion and the role of lactogenic hormones. Horm. Metab. Res. 29, 301-307 (1997).

23. Schraenen, A. et al. mRNA expression analysis of cell cycle genes in islets of pregnant mice. Diabetologia 53, 2579-2588 (2010).

24. Li, N. et al. Ablation of somatostatin cells leads to impaired pancreatic islet function and neonatal death in rodents. Cell Death Dis. 9, 682 (2018).

25. Liang, Y. L. et al. Phase-plate cryo-EM structure of a biased agonist-bound human GLP-1 receptor-Gs complex. Nature 555, 121-125 (2018).

26. $\mathrm{Hu}, \mathrm{Q}$. X. et al. Constitutive Galphai coupling activity of very large $\mathrm{G}$ proteincoupled receptor 1 (VLGR1) and its regulation by PDZD7 protein. J. Biol. Chem 289, 24215-24225 (2014).

27. Huch, M. et al. Long-term culture of genome-stable bipotent stem cells from adult human liver. Cell 160, 299-312 (2015).

28. Staels, W. et al. Conditional islet hypovascularisation does not preclude beta cell expansion during pregnancy in mice. Diabetologia 60, 1051-1056 (2017).

29. Kim, H. et al. Serotonin regulates pancreatic beta cell mass during pregnancy. Nat. Med. 16, 804-808 (2010).

30. Karnik, S. K. et al. Menin controls growth of pancreatic beta-cells in pregnant mice and promotes gestational diabetes mellitus. Science 318, 806-809 (2007)

31. Zhao, X. et al. Involvement of the STAT5-cyclin D/CDK4-pRb pathway in betacell proliferation stimulated by prolactin during pregnancy. Am. J. Physiol. Endocrinol. Metab. 316, E135-E144 (2019).

32. Lebensohn, A. M. \& Rohatgi, R. R-spondins can potentiate WNT signaling without LGRs. Elife. 7, https://doi.org/10.7554/eLife.33126 (2018). 